\title{
Efeito de um protocolo fisioterapêutico para as disfunções decorrentes das tendinopatias do ombro
}

\author{
Effect of a physiotherapy protocol for the shoulder tendinopathy disorders
}

\author{
Benjamin Ottobelli Neto ${ }^{1}$, Claudia Carolina Andrade Perea ${ }^{1}$, Fabíola Machinski ${ }^{1}$, \\ Andersom Ricardo Fréz ${ }^{2}$, João Afonso Ruaro ${ }^{3}$, Morgana Carbonera Raimondi ${ }^{4}$
}

\section{RESUMO}

Objetivo: O objetivo deste estudo foi avaliar o efeito de um protocolo de atendimento fisioterapêutico para as disfunções decorrentes das tendinopatias do ombro, sendo considerado o impacto destas lesões sobre a função, dor e mobilidade desta articulação.

Materiais e Métodos: Sete voluntários com idade entre 20 e 53 anos, com tendionpatia do ombro, foram submetidos a um protocolo fisioterapêutico durante 8 semanas, aplicado 3 vezes por semana com duração média de 45 minutos cada. O protocolo estava dividido em 3 fases. Os objetivos da fase I foram: reduzir o processo inflamatório, a dor, e aumentar a amplitude de movimento (ADM). Na fase II objetivou-se manter e/ou aumentar a flexibilidade e fortalecer os músculos do ombro. Já a fase III teve por objetivo aprimorar o desempenho muscular e ganhar propriocepção. Como métodos de avaliação foram utilizados o Questionário Western Ontario Rotador Cuff Index (WORC), a Escala Visual Análoga (EVA), o Questionário de dor McGill e mensuração da ADM, todos aplicados no início e no final do tratamento.

Resultados: A aplicação do protocolo foi eficaz no tratamento dos voluntários, apresentando melhora significativa na função $(p=0,0023)$; redução na EVA $(p=0,0142)$, no índice da dor $(p=0,0017)$ e no número de descritores da dor $(p=0,0011)$. Em relação à $A D M$, apenas a abdução do ombro não apresentou aumento significativo.

Conclusão: $O$ protocolo utilizado neste estudo apresentou resultado positivo no que diz respeito à melhora funcional, a dor e a mobilidade em pacientes com tendinopatias do ombro.

Palavras-chave: ombro; tendinopatia; modalidades em fisioterapia.

\begin{abstract}
Objective: The aim of this study was to assess the physiotherapy protocol effect in the treatment of shoulder tendinopathy disorders, considering the impact of these lesions on the function, pain and joint mobility.

Materials and Methods: A total of 7 volunteers, between the age of 20 and 53 years old, with shoulder tendinopathy, were submitted to a physiotherapy protocol for 8 weeks, applied 3 times a week, for 45 minutes each. The protocol was divided into 3 stages with different goals and modalities. Stage I was realized to reduce pain and inflammatory process and increase the range of motion (ROM). Stage II purposed to increase shoulder muscles flexibility and strength. At stage III, endurance and proprioceptive exercises were done. As evaluation methods, we have used the Western Ontario Rotator Cuff Index (WORC), the Visual Analogue Scale (VAS), the McGill Pain Questionnaire and the ROM measurement. All measures were performed at baseline and at the end of treatment.

Results: The protocol was effective in the volunteers treatment, showing a significant improvement in the function $(p=0.0023)$; and a significant reduction in the VAS $(p=0.0142)$, pain index $(p=0.0017)$ and pain descriptors number $(p=0.0011)$. Regarding the ROM, only the shoulder abduction did not significantly increased.

Conclusions: The protocol used in this study showed positive results regarding functional improvement, pain and mobility in patients with shoulder tendinopathy.
\end{abstract}

Keywords: shoulder; tendinopathy; physical therapy modalities.

\footnotetext{
${ }^{1}$ Fisioterapeuta. Graduado pela Faculdade Anglo-Americano (FAA).

${ }^{2}$ Fisioterapeuta. Mestre em Saúde, Interdisciplinaridade e Reabilitação pela Universidade Estadual de Campinas (UNICAMP). Docente do Departamento de Fisioterapia da Universidade Estadual do Centro-Oeste (Unicentro).

${ }^{3}$ Fisioterapeuta. Mestre em Engenharia Biomédica pela Universidade do Vale do Paraíba (UNIVAP). Docente do Curso de Fisioterapia da Universidade Federal do Rio Grande do Norte (UFRN), Faculdade de Ciências da Saúde do Trairi (FACISA).

${ }^{4}$ Fisioterapeuta. Especialista em Fisioterapia Traumato-Ortopédica e Desportiva pelo Instituto Brasileiro de Pesquisa e Extensão (IBPEX) e Especialista em Fisioterapia Dermato Funcional pelo Instituto Brasileiro de Therapias (IBRATE). Docente do Curso de Fisioterapia da FAA.
} 


\section{INTRODUÇÃO}

Como o ombro é uma articulação complexa é necessário que todas as estruturas estejam em sincronia para manter a biomecânica normal ${ }^{1}$. Desta forma, alterações que comprometam a estrutura e a função fazem com que esse complexo articular seja alvo de inúmeras afecções, entre elas as tendinopatias. Estas afecções podem apresentar etiologia decorrente de fatores mecânicos e vasculares ${ }^{2}$.

Entre os mecânicos destaca-se a carga repetitiva, a qual mesmo dentro da faixa de oscilação de tensão normal-fisiológica de um tendão causa fadiga e, eventualmente, leva à falência tendínea, pois há acúmulo de danos no colágeno ou em outros componentes da matriz colágena. Já para os vasculares, os tendões, tecidos metabolicamente ativos que necessitam de aporte vascular, apresentam uma provisão de sangue deficiente, deixando-os mais suscetíveis a degenerações ${ }^{2}$.

Estas disfunções no ombro geram dor e redução da mobilidade articular e, em consequência, afetam de forma negativa a capacidade funcional, as atividades laborais e a qualidade de vida das pessoas ${ }^{3}$, além de representarem um problema socioeconômico ${ }^{4}$. Estudos apontam que 14 a $21 \%$ das pessoas referem queixa de dor na região do ombro ${ }^{5,6}$, podendo chegar entre $37 \mathrm{e}$ $45 \%$ quando se estuda trabalhadores dos setores de indústria e de serviços ${ }^{7-9}$.

A fim de minimizar o impacto causado por essas lesões na vida das pessoas e na sociedade, programas de reabilitação e prevenção devem ser maximizados ${ }^{10}$, porém, previamente à recuperação funcional do ombro, torna-se necessário realizar uma avaliação estruturada que inclui a anamnese em conjunto com a realização de procedimentos de avaliação clínica, que geralmente envolvem testes. Com base na resposta destes testes clínicos, o diagnóstico de tendinopatias é alcançado ${ }^{11}$. Além disso, no início, durante e após o período de tratamento, instrumentos de medida são utilizados por fisioterapeutas e outros profissionais como referência para planejar o programa de reabilitação e para monitorar sua eficácia ${ }^{10}$.

Depois de estabelecido o diagnóstico cinéticofuncional, estas lesões podem ser submetidas ao tratamento fisioterapêutico, que objetiva atuar na regeneração e recuperação funcional dos tecidos afetados. Assim, o objetivo deste trabalho foi verificar o efeito de um protocolo de atendimento fisioterapêutico para as disfunções decorrentes das tendinopatias do ombro, sendo considerado o impacto destas lesões sobre a função, dor e mobilidade da articulação.

\section{MATERIAIS E MÉTODOS}

A presente pesquisa caracterizou-se como um estudo quasi-experimental do tipo antes e depois. A amostra foi formada por demanda espontânea, inicialmente 9 voluntários, 8 do sexo feminino e 1 do masculino, idade entre 20 e 53 anos. Duas voluntárias foram desligadas por terem mais de 3 faltas aos atendimentos, sendo a amostra constituída por 7 voluntários (4 ombros direitos e 3 esquerdos). Em relação às atividades ocupacionais, 2 voluntárias eram cabeleireiras, 1 doméstica, 1 serviços gerais, 1 estudante, 1 do lar e o voluntário era vendedor.

O estudo foi realizado na Clínica-Escola de Fisioterapia da Faculdade Anglo-Americano, em Foz do Iguaçu, Paraná. A pesquisa foi aprovada pelo Comitê de Ética em Pesquisa da Faculdade Assis Gurgacz (CEP/FAG), protocolo 187/2010. Todos os voluntários foram examinados pelo mesmo pesquisador, por meio de avaliação fisioterapêutica baseada em métodos subjetivos e objetivos, que incluíram: anamnese, avaliação da dor, amplitude de movimento (ADM) ativa e avaliação da função do complexo articular do ombro. A avaliação foi realizada no primeiro e no último atendimento.

A intensidade da dor foi mensurada de utilizando-se a Escala Visual Analógica (EVA), a qual consiste de uma linha reta de $10 \mathrm{~cm}$, não numerada, indicando-se em uma extremidade a marcação "ausência de dor" e na outra, "pior dor imaginável"12. Essa escala fornece informações conclusivas para o diagnóstico da intensidade da dor ${ }^{13}$.

Já para avaliar os aspectos multidimensionais da dor foi utilizado o Questionário de dor McGill. O questionário é formado por 78 descritores, organizado em quatro grupos (sensitivo-discriminativo, afetivo-motivacional, cognitivo-avaliativo e miscelânia) e 20 subgrupos. Esse questionário fornece dois escores: número de 
descritores escolhidos, que pode ser no máximo 20 , pois é possível escolher apenas um descritor por subgrupo; e índice de avaliação da dor, o qual corresponde ao somatório dos valores atribuídos a cada descritor escolhido no questionário, sendo o valor máximo possível $78^{14}$.

Já para a avaliação da função foi aplicada a versão brasileira do Questionário Rotator Cuff Western Ontario Questionnaire Index (WORC) ${ }^{15}$, traduzido e adaptado culturalmente para ser aplicado em pacientes com alterações do manguito rotador (MR) na população nacional, cuja utilização foi devidamente autorizado pelos autores do questionário original ${ }^{16}$, que é constituído por 21 questões, abrangendo cinco domínios: sintomas físicos, esportes e recreação, trabalho, estilo de vida, e estado emocional. O escore final pode variar de zero a 2100. Pontuação zero implica em nenhuma redução na função e 2100 indica total comprometimento funcional. Também foram avaliadas as ADMs do ombro, por meio do uso da goniometria ${ }^{17}$. Foram considerados os movimentos: flexão, extensão, abdução, adução, rotação interna e externa e abdução e adução horizontal, todos realizados ativamente.

Foi proposto um protocolo funcional para tratamento das disfunções do ombro decorrentes de tendinopatias do MR, o qual foi dividido em três fases, com objetivos e condutas diferentes, baseado em literatura relacionada ao tema ${ }^{18-20}$.

Fase I - Objetivos: reduzir o processo inflamatório, proporcionar analgesia e ganho de ADM. Conduta: ultrassom; alongamento de musculatura póstero-lateral cervical; mobilização em decúbito lateral de escápula; liberação miofascial; liberação de trigger points utilizando a técnica de dígito-pressão; mobilização intraarticular; decoaptação de cápsula; mobilização em ADM tolerável com bastões e roldanas; crioterapia por 20 minutos. Parâmetros para evolução para a fase II: aumento de goniometria em $20 \%$, em pelo menos quatro movimentos; diminuição da dor na EVA em 20\%.

Fase II - Objetivos: manter e/ou aumentar a flexibilidade e realizar fortalecimento muscular. Conduta: fortalecimentos isométrico e isotônico do $M R$; fortalecimento isotônico de extensores do ombro; fortalecimento isotônico de rombóides; fortalecimento isotônico de serrátil; flexão de braço com apoio de joelhos bilateral; movimentos de atividades de vida diária (AVDs). Parâmetros para evolução para a fase III: aumento de força muscular em uma casa conforme o teste de força (grau I, II, III, IV e V).

Fase III - Objetivos: aprimorar o desempenho muscular, ganhar propriocepção e finalizar a recuperação funcional. Conduta: exercícios proprioceptivos com a bola terapêutica, shoulder flex e na plataforma de equilíbrio; arremessos de lances livres na cesta de basquete.

Após a avaliação os voluntários iniciaram o tratamento, com duração de 8 semanas, sendo aplicado 3 vezes por semana com duração média de 45 minutos cada atendimento. Assim, cada voluntário foi submetido a 24 atendimentos.

Foi realizada análise estatística descritiva para calcular a média e o desvio padrão dos resultados. Para avaliar a normalidade dos dados foi aplicado o teste de Kolmogorov-Smirnov e a distribuição foi considerada normal se $p>0,05$. Como todos os resultados apresentaram distribuição normal foi utilizado o teste $t$ pareado para a comparação pré e pós-intervenção. 0 nível de significância foi estabelecido em $5 \%$. Os testes foram realizados com o uso do software Graphpad Instat versão 3.0 .

\section{RESULTADOS}

Escala visual analógica

Pela avaliação com a EVA foi observado redução no quadro álgico $(p=0,0142)$, tendo com média inicial

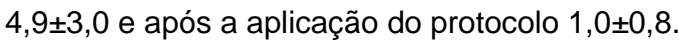

\section{Questionário de dor McGill}

Tanto a característica $(p=0,0011)$ como o índice de dor $(p=0,0017)$ apresentaram redução após a aplicação do protocolo (tabela 1).

\section{Questionário WORC}

Em relação ao impacto das tendinopatias sobre as funções do ombro, foi observada redução em todos os escores, o que representa aumento na função do membro acometido (tabela 2). 
TABELA 1 - Resultados do questionário McGill antes e após a aplicação do protocolo.

\begin{tabular}{cccc}
\hline & Avaliação inicial & Avaliação final & Valor de $\mathbf{p}$ \\
\hline Total de descritores & $12,0 \pm 3,6$ & $6,0 \pm 3,4$ & $0,0017^{*}$ \\
Sensorial & $6,0 \pm 2,5$ & $3,3 \pm 2,5$ & $0,0528^{*}$ \\
Afetivo & $2,6 \pm 1,0$ & $1,1 \pm 0,9$ & $0,0082^{*}$ \\
Avaliativo & $1,0 \pm 0$ & $1,0 \pm 0$ & - \\
Miscelânea & $2,4 \pm 0,8$ & $0,6 \pm 0,8$ & $0,0037^{*}$ \\
Total índice de dor & $24,6 \pm 8,2$ & $10,9 \pm 7,8$ & $0,0011^{*}$ \\
Sensorial & $14,6 \pm 7,9$ & $6,4 \pm 6,5$ & $0,0128^{*}$ \\
Afetivo & $3,3 \pm 1,6$ & $1,3 \pm 1,3$ & $0,0064^{*}$ \\
Avaliativo & $2,7 \pm 1,4$ & $2,0 \pm 1,2$ & 0,1824 \\
Miscelânea & $4,6 \pm 2,5$ & $1,1 \pm 1,5$ & $0,0413^{*}$ \\
\hline
\end{tabular}

*diferença significativa

\section{Amplitude de movimento}

Apenas no movimento de abdução do ombro não foi observado aumento da ADM (tabela 3).

\section{DISCUSSÃO}

Como a maioria das patologias do ombro deve ser tratada conservadoramente ${ }^{21,22}$, a intervenção fisioterapêutica, nessa situação, é considerada a primeira escolha de tratamento por melhorar o equilíbrio muscular da região, aumentar a funcionalidade do membro acometido e possibilitar retorno mais rápido às atividades de vida diária com diminuição das dores. Além disso, existem indicações seguras de que o tratamento cirúrgico não difere significantemente da intervenção fisioterapêutica quanto à melhora da funcionalidade e quanto à redução da dor $^{23,24}$. Desta forma, foi proposto um protocolo para tratamento conservador, utilizando-se como base descrições de tratamento para disfunções do ombro disponíveis na literatura.

$\mathrm{Na}$ abordagem fisioterapêutica para estas disfunções deve-se incluir crioterapia, mobilização articular, reequilíbrio neuromuscular, exercícios de fortalecimento, eletroterapia e decoaptação $\operatorname{articular}^{22}$, indicações de tratamento que corroboraram com o protocolo proposto. A conduta fisioterapêutica proposta por Ginn et al. ${ }^{25}$ para tratamento das tendinopatias de origem mecânica, apresentou melhora na dor durante a abdução e flexão do ombro, assim como na capacidade funcional e na autopercepção do indivíduo. Além disso, ao se compararem os dois grupos tratados com e sem a prescrição de exercícios, observou-se que o primeiro grupo aumentou a função do ombro ${ }^{26}$. Portanto, a fisioterapia por meio da vasta gama de recursos disponíveis é uma importante aliada no tratamento conservador, podendo proporcionar, além do alívio das condições sintomatológicas, o restabelecimento da função normal do ombro acometido.

Entre estes recursos cita-se o alongamento da unidade musculotendínea, 0 qual reduz significativamente a tensão passiva do tecido ${ }^{27}$, com conseqüente aumento da ADM. Além disso, os músculos do MR induzem à abdução da escápula, que é contrabalanceada pelos músculos rombóides e elevador da escápula e pelas fibras superiores do trapézio. Esse equilíbrio é fundamental para a adequação dos ritmos escapulotorácico e escapuloumeral durante os movimentos do membro superior, por isso foram utilizadas técnicas miofasciais para restabelecer 0 equilíbrio entre essas musculaturas e promover a melhora das amplitudes de movimentos, o que está de acordo com o observado por Souza ${ }^{28}$. Entretanto, não se pode relacionar este aumento unicamente ao alongamento e ao equilíbrio neuromuscular, pois a redução da dor também foi significativa. 
TABELA 2 - Resultados do questionário WORC antes e após a aplicação do protocolo.

\begin{tabular}{lccc}
\hline & Avaliação inicial & Avaliação final & Valor de p \\
\hline Sintomas Físicos & $353,6 \pm 96,2$ & $128,3 \pm 60,5$ & $0,0014^{*}$ \\
Esporte/Recreação & $224,1 \pm 41,4$ & $68,4 \pm 30,2$ & $0,0003^{\star}$ \\
Trabalho & $235,4 \pm 121,4$ & $105,0 \pm 50,3$ & $0,0307^{\star}$ \\
Estilo de Vida & $228,6 \pm 121,8$ & $61,1 \pm 38,0$ & $0,0056^{*}$ \\
Emoções & $165,1 \pm 95,1$ & $69,9 \pm 46,8$ & $0,0165^{\star}$ \\
Resumo & $1206,9 \pm 395,4$ & $439,9 \pm 191,0$ & $0,0023^{*}$ \\
\hline
\end{tabular}

*diferença significativa

O uso do Questionário de McGill demonstrou-se adequado e de fácil aplicação para a amostra, fato já citado por Camargo et al. ${ }^{29}$, que realizaram um estudo com 27 trabalhadores com síndrome do impacto observaram que o Questionário de dor McGill é uma ferramenta importante e útil na avaliação de diversas situações clínicas do ombro.

Já para avaliar a funcionalidade do ombro, Knaut et al. ${ }^{10}$ relataram que existem vários instrumentos de medidas voltados para esta avaliação encontrados na literatura, porém apenas alguns apresentam versões em português. Um destes instrumentos de medidas é o WORC $^{15}$, o qual foi utilizado nesta pesquisa. Além deste, mais 3 instrumentos foram traduzidos e adaptados culturalmente para a língua portuguesa: o Disabilities of the Arm, Shoulder and Hand (DASH) Questionnaire $^{30}$, o American Shoulder and Elbow Surgeons Standardized Shoulder Assessment Form $(\text { ASES })^{10}$ e o Penn Shoulder Score (PSS) ${ }^{31}$. O primeiro não foi utilizado por avaliar o membro superior de uma forma mais abrangente, não sendo específico para o ombro, enquanto os outros por terem sido publicados após o início da pesquisa. Destaca-se que para todas estas ferramentas o objetivo da sua aplicação é a padronização da avaliação do ombro e/ou membro superior.

Os resultados da presente pesquisa ainda estão de acordo com outras pesquisas que utilizaram-se do tratamento fisioterapêutico do ombro, como a pesquisa de Rayegani et al. $^{32}$, um ensaio clínico cego randomizado, que comparou o efeito do ultrassom e do laser em disfunções miofasciais do ombro e concluiu que ambos os métodos apresentam resultados positivos. Resultados satisfatórios também forma demonstrados no estudo de Hidalgo-Lozano et al. ${ }^{33}$, que pesquisaram o efeito de técnicas de terapia manual no tratamento muscular de pacientes com pinçamento no ombro, tendo achado que tais métodos reduziram a dor e a sensibilidade à pressão nos pacientes tratados.

Por fim, os resultados do presente estudo corroboram com os achados de Marinko et al. ${ }^{34}$, que realizaram uma importante metanálise sobre o efeito do exercício terapêutico em portadores de disfunções no ombro, considerando amplitude de movimento, dor e função, exatamente as variáveis avaliadas na presente pesquisa. Os autores acharam que os exercícios são uma intervenção efetiva nas condições dolorosas e função, sendo inconclusivos quanto à ADM em virtude das falhas metodológicas dos estudos pesquisados.

Este estudo limitou-se a uma amostra de pessoas de Foz do Iguaçu, Paraná, com disfunções no ombro decorrentes de tendinopatias desta articulação. O número pequeno de sujeitos limitou a formação de um grupo controle, como também reduziu o poder da análise estatística. Sugere-se a continuidade do estudo, porém de forma longitudinal, incluindo estudos de seguimento (follow up) buscando identificar melhor os fatores que interferem na qualidade de vida e no estado funcional desses pacientes, assim como na recidiva das disfunções.

Assim, o protocolo utilizado neste estudo apresentou um resultado positivo na amostra analisada, considerando-se as condições experimentais descritas, No que diz respeito à melhora funcional, a dor e a 
TABELA 3 - ADM do membro acometido antes a após a aplicação do protocolo.

\begin{tabular}{lccc}
\hline & Avaliação Inicial & Avaliação Final & Valor de p \\
\hline Flexão & $141,4 \pm 27,3$ & $170,7 \pm 7,1$ & $0,0204^{*}$ \\
Extensão & $40,0 \pm 8,3$ & $61,1 \pm 13,5$ & $0,0090^{*}$ \\
Abdução & $153,3 \pm 27,6$ & $175,6 \pm 4,8$ & 0,0875 \\
Adução & $35,9 \pm 9,6$ & $56,1 \pm 12,0$ & $0,0017^{*}$ \\
Rotação Interna & $58,6 \pm 22,8$ & $82,7 \pm 9,3$ & $0,0181^{*}$ \\
Rotação Externa & $65,0 \pm 26,1$ & $89,0 \pm 2,6$ & $0,0410^{*}$ \\
Abdução Horizontal & $81,6 \pm 16,6$ & $105,1 \pm 10,4$ & $0,0007^{*}$ \\
Adução Horizontal & $30,4 \pm 6,9$ & $54,6 \pm 6,7$ & $<0,0001^{*}$ \\
\hline
\end{tabular}

*diferença significativa

amplitude de movimento em pacientes com disfunções do ombro decorrentes das tendinopatias do manguito rotador.

\section{REFERÊNCIAS}

1. Santos OS, Bonamin C, Sobania LC, Otsuka N, Sobania RL. Síndrome do impacto: resultados do tratamento cirúrgico. Rev Bras Ortop. 1995;30(9):655-9.

2. Ejnismann B, Monteiro GC, Uyeda LF. Ombro doloroso. Rev Einstein. 2008;6(1):133-7.

3. Mousavi SJ, Parnianpour M, Abedi M, Askary-Ashtiani A Karimi A, Khorsandi $A$, et al. Cultural adaptation and validation of the Persian version of the Disabilities of the Arm, Shoulder and Hand (DASH) outcome measure. Clin Rehabil. 2008;22(8):749-57.

4. Grooten WJ, Mulder M, Josephson M, Alfredsson L, Wiktorin C. The influence of work-related exposures on the prognosis of neck/shoulder pain. Eur Spine J. 2007;16(12):2083-91.

5. Bongers PM. The cost of shoulder pain at work. BMJ. 2001;322(7278):64-5.

6. Urwin M, Symmons D, Allison T, Brammah T, Busby $H$ Roxby M, et al. Estimating the burden of musculoskeletal disorders in the community: the comparative prevalence of symptoms at different anatomical sites, and the relation to social deprivation. Ann Rheum Dis. 1998;57(11):649-55.

7. Andersen JH, Haahr JP, Frost P. Risk factors for more severe regional musculoskeletal symptoms: a two-year prospective study of a general working population. Arthritis Rheum. 2007;56(4):1355-64.

8. Cassas KJ, Cassettari-Wayhs A. Childhood and adolescent sportsrelated overuse injuries. Am Fam Physician. 2006;73(6):1014-22.

9. Eltayeb S, Staal JB, Kennes J, Lamberts PH, de Bie RA. Prevalence of complaints of arm, neck and shoulder among computer office workers and psychometric evaluation of a risk factor questionnaire. BMC Musculoskelet Disord 2007;8:68

10. Knaut LA, Moser ADL, Melo SA, Richards RR. Tradução e adaptação cultural à língua portuguesa do American Shoulder and Elbow Surgeons Standardized Shoulder Assessment Form (ASES) para avaliar a função do ombro. Rev Bras Reumatol. 2010;50(2):176-89.
11. Lewis JS. Rotator cuff tendinopathy/subacromial impingement syndrome: is it time for a new method of assessment? Br J Sports Med. 2009;43(4):259-64.

12. Teixeira MJ, Pimenta CAM. Avaliação do doente com dor. In: Teixeira MJ, Figueiró JAB. Dor: epidemiologia, fisiopatologia, avaliação, síndromes dolorosas e tratamento. São Paulo: Moreira Jr; 2001. p. 14-35.

13. Rebellatto JR, Morelli JGS. Fisioterapia geriátrica: a prática da assistência ao idoso. Barueri: Manole; 2004.

14. Pimenta CAM, Teixeira MJ. Questionário de dor McGill: proposta de adaptação para a língua portuguesa. Rev Esc Enferm USP. 1996;30(3):473-83.

15. Lopes AD, Stadniky SP, Masiero D, Carrera EF, Ciconelli RM, Griffin S. Tradução e adaptação cultural do WORC: um questionário de qualidade de vida para alterações do manguito rotador. Rev Bras Fisioter. 2006;10(3):309-15.

16. Kirkley A, Alvarez C, Griffin S. The development and evaluation of a disease-specific quality-of-life questionnaire for disorders of the rotator cuff: The Western Ontario Rotator Cuff Index. Clin J Sport Med. 2003;13(2):84-92.

17. Marques AP. Manual de goniometria. $2^{\underline{a}}$ ed. Barueri: Manole; 2003.

18. Hall CM, Brody LT. Exercício terapêutico na busca da função. $2^{\mathrm{a}}$ ed. Rio de Janeiro: Guanabara Koogan; 2007.

19. Hertling D, Kesller MR. Tratamento de distúrbios musculoesqueléticos comuns: princípios e métodos de fisioterapia. 4 ${ }^{\mathrm{a}}$ ed. Barueri: Manole; 2009.

20. Kisner C, Colby A. Exercícios terapêuticos: fundamentos e técnicas. $5^{\mathrm{a}}$ ed. Barueri: Manole; 2010.

21. Morelli RSS, Vulcano DR. Princípios e procedimentos utilizados na reabilitação das doenças do ombro. Rev Bras Ortop. 1993;28(9):653-6.

22. Giordano M, Giordano V, Giordano LHB, Giordano JN Tratamento conservador da síndrome do impacto subacromial: estudo em 21 pacientes. Acta Fisiatr. 2000;7(1):13-9.

23. Haar JP, Østergaard S, Dalsgaard J, Norup K, Frost P, Lausen $S$, et al. Exercises versus arthroscopic descompression in patients with subacromial impingement: a randomised, controlled study in 90 cases with a one year follow up. Ann Rheum Dis. 2005;64(5):760-4.

24. Lima GCS, Barbosa EM, Alfieri FM. Análise da funcionalidade e da dor e de indivíduos portadores de síndrome do impacto, submetidos à intervenção 
fisioterapêutica. Fisioter Mov. 2007;20(1):61-9.

25. Ginn KA, Hebert RD, Khouw W, Lee R. A randomized, controlled clinical trial of a treatment for shoulder pain. Phys Ther. 1997;77(8):802-11.

26. Howell S, Imobersteg A, Seger D, Marone P. Classification of the role of the supraspinatus muscle in shoulder function. J Bone Joint Surg. 1986;68A:398-404.

27. Magnusson SP. Passive properties of human skeletal muscle during stretch maneuvers: a review. Scand J Med Sci Sports. 1998;8(2):65-77

28. Souza MZ. Reabilitação do complexo do ombro. São Paulo: Manole; 2001.

29. Camargo PR, Haik MN, Filho RB, Mattiello RSM, Salvini TF. Pain in workers with shoulder impingement syndrome: an assessment using the DASH and McGill pain questionnaires. Rev Bras Fisioter. 2007;11(2):161-7.

30. Orfale AG, Araújo PMP, Ferraz MB, Natour J. Translation into Brazilian Portuguese, cultural adaptation and evaluation of the reliability of the disabilities of the arm, shoulder and hand questionnaire. Braz J Med Biol Res. 2005;38:293-302.

31. Napoles BV, Hoffman CB, Martins J, Oliveira AS. Tradução e adaptação cultural do Penn Shoulder Score para a língua portuguesa: PSS-Brasil. Rev Bras Reumatol. 2010;50(4):389-407.
32. Rayegani S, Bahrami M, Samadi B, Sedighipour L, Mokhtarirad M, Eliaspoor D. Comparison of the effects of low energy laser and ultrasound in treatment of shoulder myofascial pain syndrome: a randomized single-blinded clinical trial. Eur J Phys Rehabil Med. 2011;47(3):381-9.

33. Hidalgo-Lozano A, Fernández-de-Las-Peñas C, DíazRodríguez L, González-Iglesias J, Palacios-Ceña D, Arroyo-Morales M. Changes in pain and pressure pain sensitivity after manual treatment of active trigger points in patients with unilateral shoulder impingement: a case series. J Bodyw Mov Ther. 2011;15(4):399-404.

34. Marinko LN, Chacko JM, Dalton D, Chacko CC. The effectiveness of therapeutic exercise for painful shoulder conditions: a meta-analysis. J Shoulder Elbow Surg. $2011 ; 20(8): 1351-9$.

\section{Endereço para correspondência}

Morgana Carbonera Raimondi Avenida Paraná 5661, Vila A

Foz do Iguaçu/PR - CEP 85868-030

Telefone: +55 4599374517

E-mail: mor.carbonera@gmail.com 